\title{
Equilibrium Configurations for a Floating Drop
}

\author{
Alan Elcrat, Robert Neel ${ }^{1}$ and David Siegel ${ }^{2}$ \\ Communicated by R. Finn
}

\begin{abstract}
If a drop of fluid of density $\rho_{1}$ rests on the surface of a fluid of density $\rho_{2}$ below a fluid of density $\rho_{0}, \rho_{0}<\rho_{1}<\rho_{2}$, the surface of the drop is made up of a sessile drop and an inverted sessile drop which match an external capillary surface. Solutions of this problem are constructed by matching solutions of the axisymmetric capillary surface equation. For general values of the surface tensions at the common boundaries of the three fluids the surfaces need not be graphs and the profiles of these axisymmetric surfaces are parametrized by their tangent angles. The solutions are obtained by finding the value of the tangent angle for which the three surfaces match. In addition the asymptotic form of the solution is found for small drops.
\end{abstract}

Mathematics Subject Classification (2000). 76B45, 35A15, 35R35, 49J05.

Keywords. Capillary surfaces, floating drop, surface tension.

\section{Introduction}

If a drop of fluid rests on the boundary between two other fluids, e.g. oil on a water surface below air, one can ask what the equilibrium shape of the drop is. This is a problem of intrinsic interest, and it has been studied experimentally and approximately, but a careful mathematical analysis has not been done. In this work we make a beginning on doing this.

Since capillary forces must be taken into account it is natural to formulate this question in terms of a variational problem for the interfacial surfaces as has been done for other capillary problems [1]. When this is done an energy functional is obtained for the surfaces of the top and bottom of the drop and the remaining surface of the lower fluid. In the second section of this paper a careful derivation is given of the equilibrium conditions for an extremal of this energy. This equilibrium condition consists of a system of three differential equations together with joining conditions where the solutions of these equations intersect, that is where

\footnotetext{
1 Supported in part by a Fulbright Scholarship and an NSF Graduate Research Fellowship.
}

2 Supported in part by an NSERC Research Grant. 
the surfaces meet. In the rest of the paper we study axisymmetric solutions, and the resulting problem may be thought of as a free boundary problem for a system of capillary surfaces. The top of the drop is a portion of a sessile drop, the bottom is a capillary surface or an extension that may bend over, and the lower fluid surface is an exterior capillary surface or its extension to an unbounded liquid bridge. In analyzing this differential equation problem we have made essential use of the work of Finn ([1], chapters 2,3) and Siegel [10] on capillary surfaces and Vogel [13] on unbounded, axisymmetric liquid bridges. A notable feature of our results is that none of the three surfaces are required à priori to be a graph.

The remainder of the paper is organized as follows. In section 3 we give some preliminary results on symmetric interior and exterior capillary surfaces. These were first studied by Johnson and Perko [5]. From results of Finn [1] we deduce the existence and uniqueness of an interior capillary surface which has a given inclination angle at a given radius (Theorem 3.1). Extending the work of Vogel [13] we obtain the existence and uniqueness of an exterior capillary surface which has a given inclination angle at a given radius (Theorem 3.2). The uniqueness part of Theorem 3.2 requires a volume comparison argument. In Section 4 we prove the existence of a drop of a given radius by a continuity method (Theorem 4.1). When $0 \leq \gamma_{02} \leq \pi / 2, \gamma_{02}$ the contact angle between the top and bottom surfaces, we show that the drop of a given radius is unique and that for every prescribed volume there is a drop with that volume (Theorem 4.2). The uniqueness comes from monotonicity properties of nonparametric interior and exterior capillary surfaces due to Finn and to Siegel, see [1] and [10]. The existence and uniqueness of a drop of a given radius in this case was initially presented by S. T. Gibbs [2]. Then we give an asymptotic result for small drops. The inclination angle of the bottom surface tends to a specific limit as the radius tends to 0. In Section 5 we consider the floating "bubble" in which the densities of the top fluid and the drop are the same, the top is a section of a sphere and the top and bottom are tangent to each other. We prove that there is a unique bubble of given radius and that for every prescribed volume there is a bubble with that volume (Theorem 5.3 and Corollary 5.1). The inclination angle of the bottom surface tends to $\pi$ as the radius tends to 0 (Theorem 5.2). Open questions are stated in Section 6.

\section{A variational problem}

A variational formulation for the equilibrium configuration of three immiscible fluids in a closed container acted upon by a conservative force field was first given by L. A. Slobozhanin [11]. We will give a version of his argument using perturbations that are graphs and where the joining condition on the contact curve is derived variationally.

Consider three immiscible fluids in a vertical cylindrical container with gravity acting downwards. The fluids, labeled with subscripts $0,1,2$, occupy regions 
$\Omega_{0}, \Omega_{1}, \Omega_{2}$. Their volumes $V_{0}, V_{1}, V_{2}$ are prescribed. Their densities are $\rho_{0}, \rho_{1}, \rho_{2}$ with $\rho_{0}<\rho_{1}<\rho_{2}$. The three interfaces between fluids $S_{01}, S_{12}, S_{02}$ will have heights $u, v, w$, respectively, and are not assumed to be graphs. We suppose that a drop of fluid 1 is formed between fluids 0 and 2, above and below the drop, that all three surfaces meet along a curve $\Gamma$ and that the interface $S_{02}$ extends from $\Gamma$ to the vertical wall of the cylinder. See Fig. 1 . The mean curvatures of

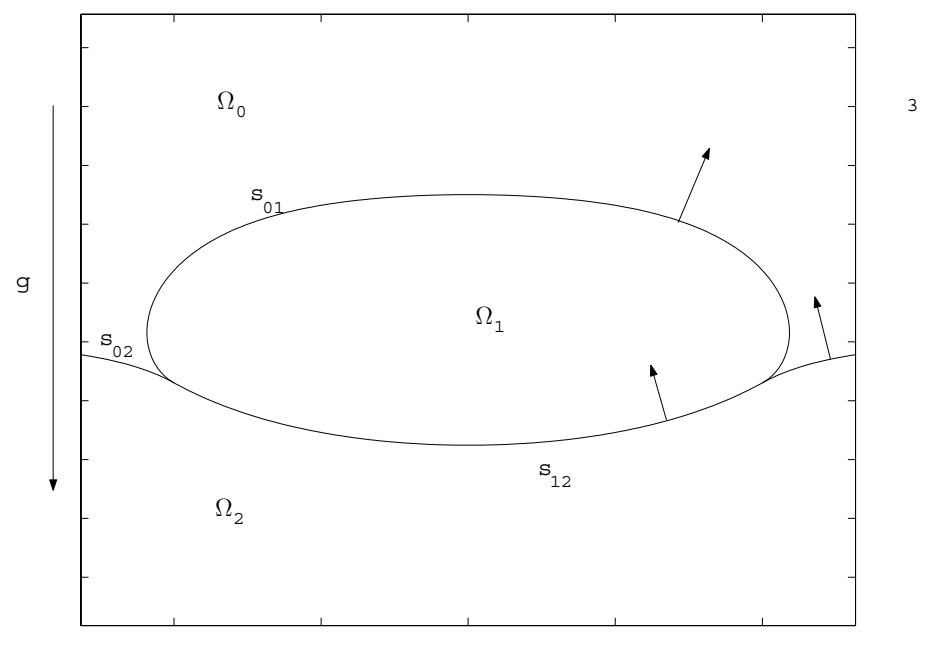

FIG. 1. Drop configuration.

the three interfaces will be denoted by $H_{u}, H_{v}, H_{w}$, with respect to an upward pointing normal, as indicated in Fig. 1. The prescribed surface tensions for the interfaces $S_{01}, S_{12}, S_{02}$ are $\sigma_{01}, \sigma_{12}, \sigma_{02}$ and the surface tension between fluid 0 (2) and the cylinder is $\sigma_{03}\left(\sigma_{23}\right)$. Let $\left|S_{i j}\right|$ be the area of $S_{i j}$ for $j<3$ and let it be the area between fluid $i$ and the container when $i=0,2$ and $j=3$. The potential energy is then

$$
E=\sum \sigma_{i j}\left|S_{i j}\right|+\sum \Phi_{i}
$$

where $\Phi_{i}=\rho_{i} g \iiint_{\Omega_{i}} z d V$ is the gravitational potential energy of the fluid $i$, where $z$ is a vertical coordinate and $g$ is the acceleration due to gravity. The two fluid case is treated in the monograph of R. Finn [1]. Let $x, y$ be coordinates in the horizontal plane. We derive conditions for an equilibrium configuration by requiring that the energy $E$ be stationary under perturbations which do not change any of the prescribed volumes. The argument proceeds in four steps.

Step 1. Near an interior point $p$ on $S_{01}$ introduce local coordinates $\bar{x}, \bar{y}, \bar{z}$ where the $\bar{x} \bar{y}$-plane is tangent to $S_{01}$ at $p$ and $\bar{z}$ is in the upward direction. See Fig. 2.

Near $p, S_{01}$ is a graph, $\bar{z}=\bar{u}(\bar{x}, \bar{y})$ for $(\bar{x}, \bar{y}) \in B, B$ a ball centered at $p$. The vertical height is denoted $u$, so that $u=u(\bar{x}, \bar{y})$. Consider a perturbation 


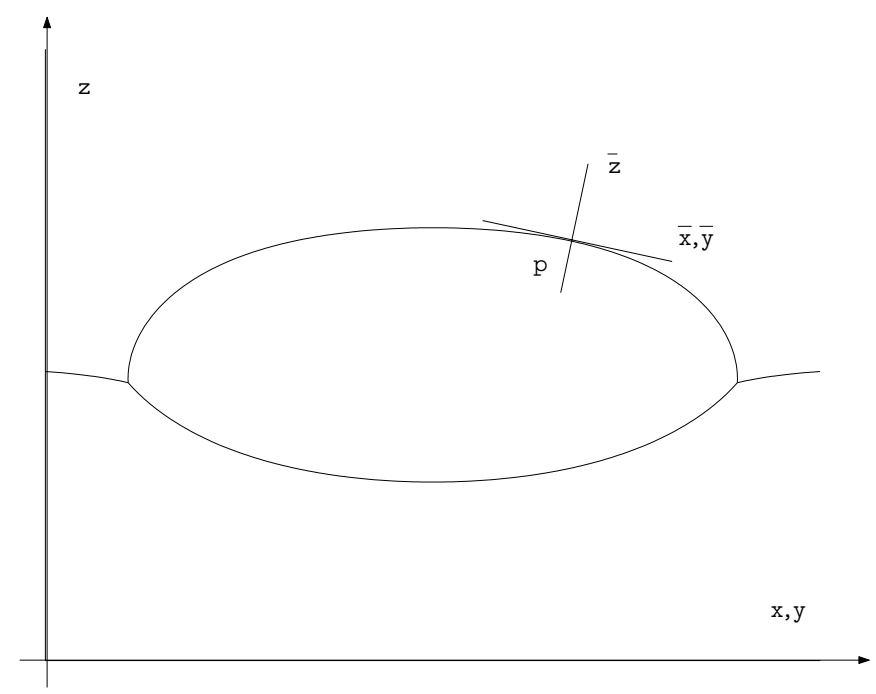

FIG. 2. Horizontal and vertical coordinates and local coordinates.

$\eta \in C_{0}^{2}(B)$ with $\iint_{B} \eta d A=0$. We have

$$
\begin{aligned}
\delta E & =\iint_{B}\left[\sigma_{01} T \bar{u} \cdot \nabla \eta+\left(\rho_{1}-\rho_{0}\right) g u \eta\right] d A \\
& =\iint_{B}\left[-\sigma_{01} \nabla \cdot(T \bar{u})+\left(\rho_{1}-\rho_{0}\right) g u\right] \eta d A
\end{aligned}
$$

where $T \bar{u}=\nabla \bar{u} / \sqrt{1+|\nabla \bar{u}|^{2}}, \nabla \cdot(T \bar{u})=2 H_{u}$. So that $\delta E=0$ implies $-2 \sigma_{01} H_{u}+$ $\left(\rho_{1}-\rho_{0}\right) g u=\lambda_{1}, \lambda_{1}$ a constant. This condition holds on all of $S_{01}$ with the same constant $\lambda_{1}$ since we assume that $S_{01}$ is connected. Similarly,

$$
\begin{aligned}
-2 \sigma_{12} H_{v}+\left(\rho_{2}-\rho_{1}\right) g v & =\lambda_{2} & & \text { on } S_{12} \\
-2 \sigma_{02} H_{w}+\left(\rho_{2}-\rho_{0}\right) g w & =\lambda_{3} & & \text { on } S_{02}
\end{aligned}
$$

where $\lambda_{2}, \lambda_{3}$ are constants.

Step 2. Let $p \in \Gamma$, where $\Gamma$ is the contact curve where the three interfaces meet. Choose a local coordinate system so that near $p$ each surface is a graph:

$$
\left\{\begin{array}{l}
\bar{z}=\bar{u}(\bar{x}, \bar{y}) \quad \text { in } B_{1}, \bar{z}=\bar{w}(\bar{x}, \bar{y}) \text { in } B_{2} \\
\bar{z}=\bar{v}(\bar{x}, \bar{y})
\end{array}\right.
$$

where $B=B_{1} \cup B_{2}, B$ is a ball about $p$. Let $\Gamma^{\prime}$ be the projection of $\Gamma$ in the $\bar{x} \bar{y}$-plane and let $\vec{\nu}$ be the unit normal on $\Gamma^{\prime}$ pointing out of $B_{1}$. See Fig. 3. The argument that we will present is similar if instead of $\bar{z}=\bar{v}(\bar{x}, \bar{y})$ in $B_{1}$ we have $\bar{z}=\bar{v}(\bar{x}, \bar{y})$ in $B_{2}$. Now consider a perturbation $\eta \in C_{0}^{2}(B)$ with $\iint_{B_{1}} \eta d A=0$, 


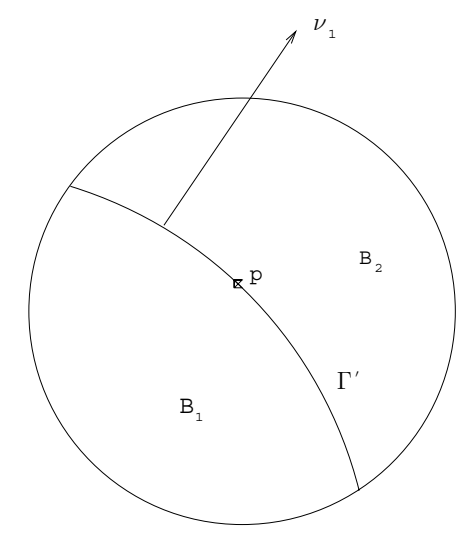

FIG. 3. Local coordinate domains.

$\iint_{B_{2}} \eta d A=0$ so that the prescribed volumes are unchanged. We have

$$
\delta E=\int_{\Gamma^{\prime}}\left[\sigma_{01} T \bar{u}+\sigma_{12} T \bar{v}-\sigma_{02} T \bar{w}\right] \cdot \vec{\nu}_{1} \eta d s .
$$

The upward normal on $S_{01}$ is $\vec{n}_{u}=\left(-T \bar{u}, 1 / \sqrt{1+|\nabla \bar{u}|^{2}}\right)$ near $p$, and similarly for $S_{12}$ and $S_{02}$. Let $\vec{\nu}=\left(\vec{\nu}_{1}, 0\right)$. $\delta E=0$ implies $\left(\sigma_{01} \vec{n}_{u}+\sigma_{12} \vec{n}_{v}-\sigma_{02} \vec{n}_{w}\right) \cdot \vec{\nu}=0$ at $p$. The vectors $\vec{n}_{u}, \vec{n}_{v}, \vec{n}_{w}, \vec{\nu}$ are coplanar since they are all orthogonal to $\Gamma$ at $p$. If we rotate our local coordinate system slightly around the tangent direction to $\Gamma$ at $p$ we obtain the same condition with a $\vec{\nu}^{*}$ close to $\vec{\nu}$ but not a multiple of $\vec{\nu}$. This implies $\sigma_{01} \vec{n}_{u}+\sigma_{12} \vec{n}_{v}-\sigma_{02} \vec{n}_{w}=\overrightarrow{0}$ at $p$ and so this condition holds on $\Gamma$.

An alternative form of this condition is $\sigma_{01} \vec{e}_{u}+\sigma_{12} \vec{e}_{v}-\sigma_{02} \vec{e}_{w}=\overrightarrow{0}$ on $\Gamma$ where $\vec{e}_{u}$ is a unit vector on $\Gamma$, orthogonal to $\Gamma$, tangent to $S_{01}$ and in the direction of $S_{01} ; \vec{e}_{v}$ and $\vec{e}_{w}$ are defined similarly. See Fig. 4 .

The alternate form follows since $\vec{e}_{u}, \vec{e}_{v}$, and $\vec{e}_{w}$ are obtained by rotating $\vec{n}_{u}, \vec{n}_{v}$, and $-\vec{n}_{w}$ counterclockwise about $\Gamma$. This known condition is interpreted as force balance along $\Gamma$, see [6] for example. Expressed in terms of contact angles this becomes

$$
\frac{\sin \gamma_{01}}{\sigma_{01}}=\frac{\sin \gamma_{02}}{\sigma_{02}}=\frac{\sin \gamma_{12}}{\sigma_{12}}
$$

where the contact angles along $\Gamma$ are indicated in Fig. 5. $\gamma_{02}$ is the contact angle between $S_{01}$ and $S_{02}$, etc.

Step 3. Next perturb all the surfaces simultaneously with $\eta_{01}, \eta_{12}, \eta_{02}, \eta_{i j} \in$ $C_{0}^{2}\left(B_{i j}\right)$, where $\iint_{B_{01}} \eta_{01} d A=\iint_{B_{12}} \eta_{12} d A=-\iint_{B_{02}} \eta_{02} d A \neq 0$, so that the volumes of the fluids are unchanged. $\delta E=0$ implies $\iint_{B_{01}} \lambda_{1} \eta_{01} d A=\iint_{B_{12}} \lambda_{2} \eta_{12} d A=$ $-\iint_{B_{02}} \lambda_{3} \eta_{02} d A=0$, giving $\lambda_{1}+\lambda_{2}-\lambda_{3}=0$.

Step 4. Finally, the surface $S_{02}$ meets the wall of the cylinder along the curve $\Gamma_{0}$. Let $p \in \Gamma_{0}$. Near $p, z=w(x, y)$ for $(x, y) \in B_{1}$ where $B_{1}$ is a portion of a 

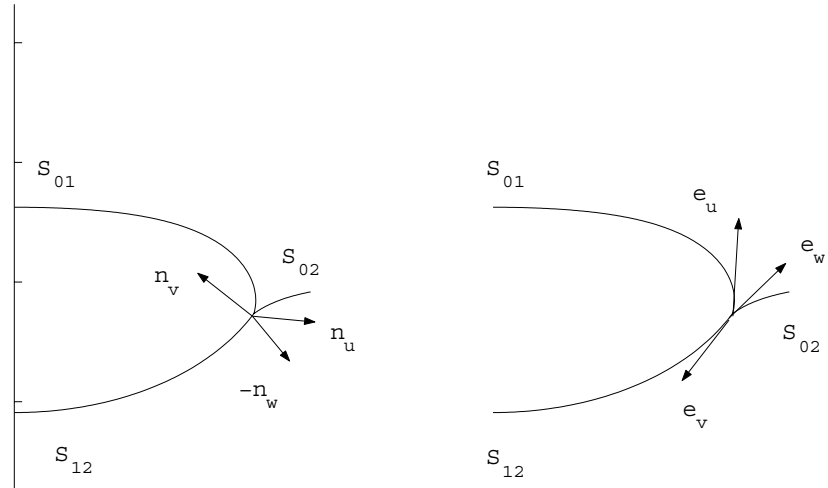

FIG. 4. Normal vectors and tangent vectors on $\Gamma$.

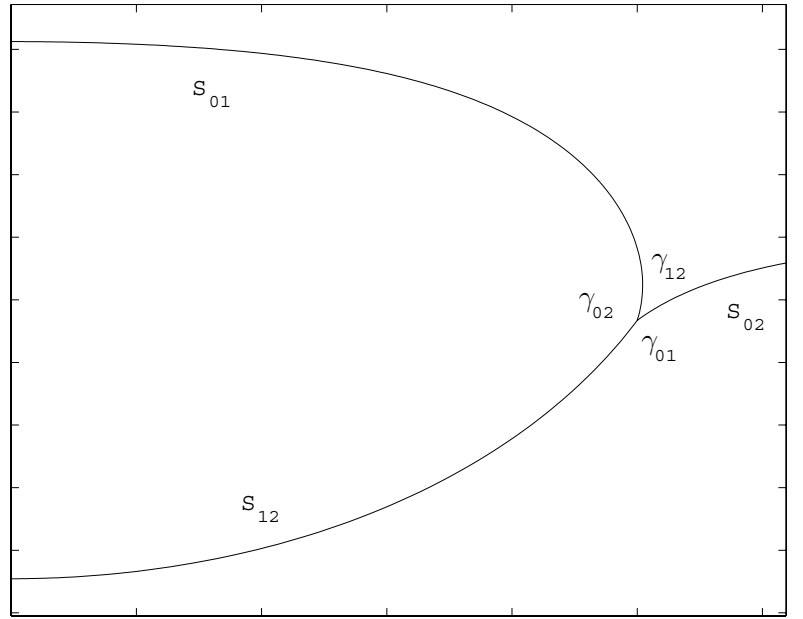

FIG. 5. Contact angles along $\Gamma$.

ball $B$ about $p$. Let $\Gamma_{0}^{\prime}$ be the projection of $\Gamma_{0}$ onto the $x y$-plane. See Fig. 6 . Let $\vec{\nu}_{1}$ be the unit normal on $\Gamma_{0}^{\prime}$ pointing out of $B_{1}$. Now perturb with $\eta \in C_{0}^{2}(B)$, $\iint_{B_{1}} \eta d A=0$.

$$
\delta E=\int_{\Gamma_{0}^{\prime}}\left[\sigma_{02} T w \cdot \vec{\nu}_{1}+\left(\sigma_{23}-\sigma_{03}\right)\right] \eta d s
$$

Since $\eta$ can be arbitrary on $\Gamma_{0}^{\prime}, \delta E=0$ implies $\sigma_{02} T w \cdot \vec{\nu}_{1}=\sigma_{03}-\sigma_{23}$ or $\cos \gamma_{0}=$ $\left(\sigma_{03}-\sigma_{23}\right) / \sigma_{02}$ where $\gamma_{0}$ is the contact angle in fluid 2 at $\Gamma_{0}$. This last argument 


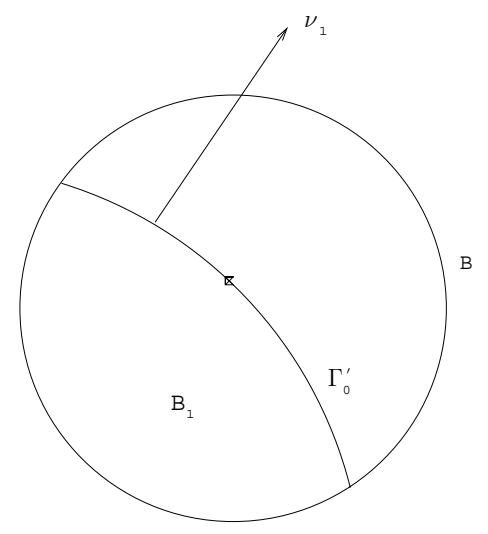

FIG. 6. The ball $B$ and domain $B_{1}$.

is completely standard, see [1].

Next consider a finite drop between infinite media. This may be considered a limiting case of the finite container. Suppose $w \rightarrow 0$ as $r \rightarrow \infty\left(r=\sqrt{x^{2}+y^{2}}\right)$. Then we must have $\lambda_{3}=0$. Let $\lambda=\lambda_{1} . \kappa_{i j}=\left(\rho_{j}-\rho_{i}\right) g / \sigma_{i j}$ (capillary constants). Define the operator $M$ to be twice the mean curvature operator so that $M u=2 H_{u}$. We have

$$
\left\{\begin{array}{l}
M u=k_{01} u+\lambda / \sigma_{01} \\
M v=\kappa_{12} v-\lambda / \sigma_{12} \\
M w=\kappa_{02} w
\end{array}\right.
$$

$u, v, w$ meet at $\Gamma$ and satisfy the force balance condition.

In the rest of this paper we will consider axisymmetric solutions to (2)-(3). In this case the contact curve $\Gamma$ will be a circle of radius $\bar{r}$, which we call the radius of the drop. We do not assume that the surfaces are graphs. The assumption on the densities implies that $\kappa_{01}, \kappa_{12}, \kappa_{02}>0$ so that $u$ and $v$ are symmetric interior capillary surfaces and $w$ is a symmetric exterior capillary surface. Let $\bar{\psi}$ denote the inclination angle of $v$ at $\bar{r}$.

Hartland and Hartley [3] have calculated drops in the special case $\rho_{1}=\rho_{2}$, $\sigma_{01}+\sigma_{02}=\sigma_{12}$ or $\sigma_{01}=\sigma_{02}=\sigma_{12} / 2$. The second case is transformable to the floating bubble case considered in section $5[(-u,-v,-w)$ satisfies the floating bubble equations]. Floating drops are studied experimentally in the papers of Princen $[7,8,9]$.

\section{Preliminaries}

We need two results on existence and uniqueness of capillary surfaces. They may also be interpreted in terms of sessile drops and liquid bridges. Consider first an axisymmetric capillary surface with center height $u_{0}$. Following Finn [1] we can 
represent this surface using the tangent angle $\psi$ as a parameter, and the surface is given by solving the differential equations

$$
\frac{d r}{d \psi}=\frac{r \cos \psi}{\kappa r u-\sin \psi}, \quad \frac{d u}{d \psi}=\frac{r \sin \psi}{\kappa r u-\sin \psi}
$$

with $r(0)=0, u(0)=u_{0}$. The surface can only be thought of as a graph $u=u(r)$ for $\psi \in[0, \pi / 2]$, but it is essential for us to use this representation for $\psi \in[0, \pi]$. If the surface is turned over it represents the profile of a sessile drop and this parameterization was used [1, chapter 3] to prove the existence of a unique sessile drop for every prescribed contact angle and volume.

Theorem 3.1. For every $\bar{r}>0$ and $\bar{\psi} \in(0, \pi]$ there is a unique solution of (4) such that $r(\bar{\psi})=\bar{r}$.

Proof. Since solutions of (4) may be thought of as $r=r\left(\psi ; u_{0}\right), u=u\left(\psi ; r_{0}\right)$ we must show that there is a unique $u_{0}>0$ such that $r\left(\bar{\psi} ; u_{0}\right)=\bar{r}$.

It has been proven in $\left[1\right.$, Theorem 3.2] that $\partial r / \partial u_{0}<0$.

We introduce the notation $R=r\left(\pi / 2 ; u_{0}\right), a=r\left(\pi ; u_{0}\right)$. Since $R<2 / \kappa u_{0}$ we have $R \rightarrow 0$ as $u_{0} \rightarrow \infty$. From [1, 2.63], $R \rightarrow \infty$ as $u_{0} \rightarrow 0$. We can now deduce that $r\left(\bar{\psi} ; u_{0}\right) \rightarrow 0$ as $u_{0} \rightarrow \infty$ and $r\left(\bar{\psi} ; u_{0}\right) \rightarrow \infty$ as $u_{0} \rightarrow 0$. The first statement follows from $r\left(\bar{\psi} ; u_{0}\right) \leq R$. For the second, if $\psi \leq \pi / 2$ we can use [1, 2.60]. For $\psi>\pi / 2$, we use $a \leq r\left(\bar{\psi} ; u_{0}\right) \leq R$ and $a \rightarrow \infty$ as $u_{0} \rightarrow 0$ which follows from [1, 3.91].

The theorem follows from the monotonicity of $r$ with respect to $u_{0}$.

We may interpret this theorem as saying there is a unique sessile drop with a given diameter and contact angle.

For the exterior surfaces there is an analogous representation [13]

$$
\frac{d u}{d \phi}=\frac{-r \sin \phi}{\kappa r u+\sin \phi}, \quad \frac{d r}{d \phi}=\frac{-r \cos \phi}{\kappa r u+\sin \phi}
$$

where $\phi$ is the tangent angle, taken as acute on the "top" of the curve. Johnson and Perko [5] have shown that for each $\sigma>0$ there is a unique solution with vertical tangent at $\sigma, r \rightarrow \infty, u \rightarrow 0$ as $\phi \rightarrow \pi$, with $u=u(r)$. The above representation allows us to conveniently extend this curve to $[0, \pi)$. We denote by $T(\sigma)$ the height at $\sigma$, i.e. $T(\sigma)=u(\pi / 2)$. The parameter $\sigma$ plays a role for exterior surfaces analogous to that $u_{0}$ plays for interior ones. It has been shown by Siegel [10] that $T(\sigma)$ is strictly increasing in $\sigma$, Vogel [13] has shown that $r(0 ; \sigma) \rightarrow 0$ as $\sigma \rightarrow 0$, and Turkington [12] has shown that $T(\sigma) \sim \sigma \ln (1 / \sigma)$ as $\sigma \rightarrow 0$.

Our next result says that there is a unique unbounded symmetric liquid bridge wetting a disk of given diameter with given contact angle [13].

Theorem 3.2. For every $\bar{r}>0$ and $\bar{\phi} \in[0, \pi)$ there is a unique solution of (5) with $r(\pi / 2 ; \sigma)=\sigma, u(\pi / 2 ; \sigma)=T(\sigma)$ such that $r(\bar{\phi} ; \sigma)=\bar{r}$. 


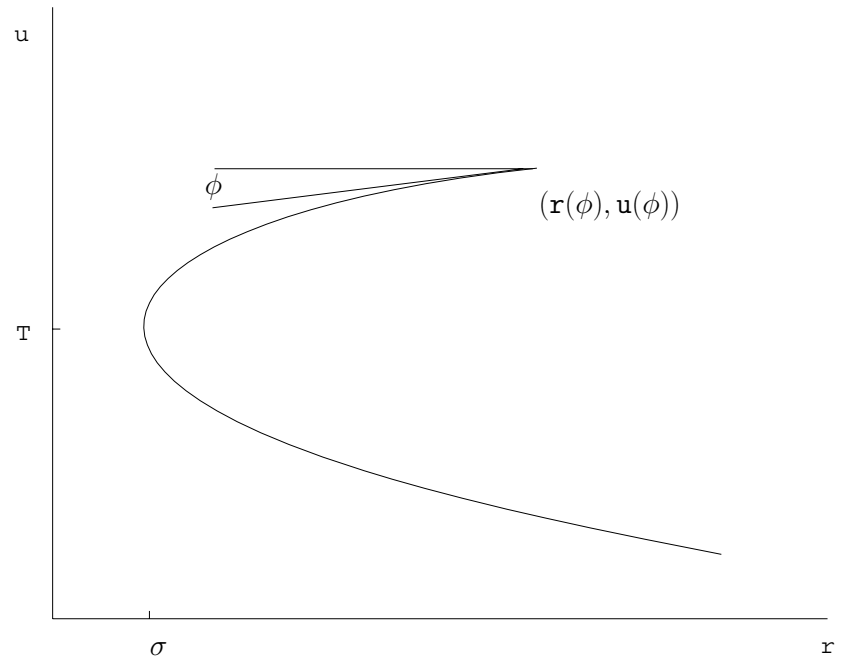

FIG. 7. Exterior surface with tangent angle $\phi$.

For $\bar{\phi} \in[\pi / 2, \pi]$ this was proven in [10]. For $\hat{\phi} \in[0, \pi / 2)$ we need only observe $\sigma \leq r(\hat{\phi} ; \sigma) \leq r(0 ; \sigma)$ and use continuity of solutions of (5) with respect to initial conditions to deduce existence.

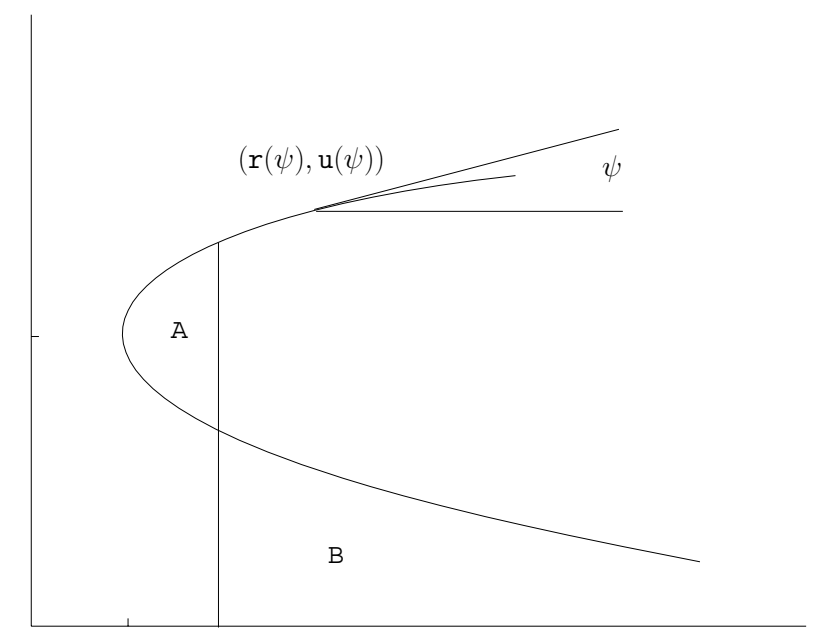

FIG. 8. A and B regions.

The proof of uniqueness is considerably more involved. We need the following lemmas due to Vogel [13]. In order to conform to his notation and to simplify 
formulas we change the scale so that we may take $\kappa=1$ in this proof.

Lemma 3.1. Let $\Gamma=(r(\phi), u(\phi))$ be a particular profile curve. Pick $\phi_{0} \in[0, \pi)$, and let $r_{0}=r\left(\phi_{0}\right)$. Let $A$ be the solid obtained by rotating the region bounded by $r=r_{0}$ and $\Gamma$ and let $B$ be the solid obtained by rotating the unbounded region between $\Gamma$ and the $r$-axis from $r=r_{0}$ to $r=+\infty$ around the $u$ axis. Then

$$
|B|-|A|=2 \pi r_{0} \sin \phi_{0} .
$$

Lemma 3.2. Let $\Gamma_{1}$ and $\Gamma_{2}$ be profile curves as above. If for some $\phi_{0} \in[0, \pi)$ we have $u\left(\phi_{0}\right)_{1}=u\left(\phi_{0}\right)_{2}$, then $\Gamma_{1} \equiv \Gamma_{2}$.

Lemma 3.3. No two distinct profile curves can cross twice.

Proof (of uniqueness). We need to show that if $\Gamma_{1}$ and $\Gamma_{2}$ are two profile curves as above with $r\left(\phi_{0}\right)_{1}=r\left(\phi_{0}\right)_{2}, \phi_{0} \in[0, \pi / 2)$, that $\Gamma_{1}$ and $\Gamma_{2}$ coincide.

Suppose that there are two such curves. Let $\sigma_{1}, \sigma_{2}$ be the radii at which they are vertical. We may assume that $u\left(\phi_{0}\right)_{1}>u\left(\phi_{0}\right)_{2}$. Suppose that $\phi_{0}$ is the largest value of $\phi$ such that $r(\phi)_{1}=r(\phi)_{2}=\rho_{0}$. (These are the leftmost such points. i.e. $\rho_{0}$ is the smallest radius.) Let $A_{1}, B_{1}, A_{2}, B_{2}$ be the volumes in Lemma 3.1 for these two curves.

We consider the intersection points of these curves with $r=\rho_{0}$. Denote by $\alpha_{U}=u\left(\phi_{0}\right)_{1}, \beta_{U}=u\left(\phi_{0}\right)_{2}$ the upper intersection heights $\left(\alpha_{U}>\beta_{U}\right)$ and $\alpha_{L}, \beta_{L}$ the lower intersection heights.

Case 1. $\alpha_{L} \leq \beta_{L}$. We have then $\alpha_{U}>\beta_{U}>\beta_{L} \geq \alpha_{L}$. Since $\Gamma_{1}$ and $\Gamma_{2}$ cannot cross more than once they cannot cross at all, so that $\Gamma_{1}$ lies entirely outside the region bounded by $\Gamma_{2}$ and $r=\rho_{0}$. It follows that $\sigma_{1}<\sigma_{2}$ and $T\left(\sigma_{1}\right)<T\left(\sigma_{2}\right)$, which implies there is a $\phi \in\left(\phi_{0}, \pi / 2\right)$ where $u(\phi)_{1}=u(\phi)_{2}$ contradicting Lemma 3.2 , and Case 1 cannot happen.

Case 2. $\alpha_{L}>\beta_{L}$. As above $T\left(\sigma_{1}\right) \leq T\left(\sigma_{2}\right)$ implies a contradiction to Lemma 3.2 , so $T\left(\sigma_{1}\right)>T\left(\sigma_{2}\right)$ and $\sigma_{1}>\sigma_{2}$. We will show that the result of Lemma 3.1 is contradicted. We begin by showing that $\left|B_{1}\right|>\left|B_{2}\right|$. First consider what happens when $\alpha_{L}<\beta_{U}$. Then $\Gamma_{1}$ and $\Gamma_{2}$ must cross somewhere above $\alpha_{L}$. Thus they cannot cross again below $\alpha_{L}$ and since $\alpha_{L}>\beta_{L}$ this implies that the lower portion of $\Gamma_{1}$ for $r>\rho_{0}$ lies completely above the corresponding portion of $\Gamma_{2}$. Thus it follows that $\left|B_{1}\right|>\left|B_{2}\right|$. Now suppose $\alpha_{L}>\beta_{U}$. Then the part of $\Gamma_{1}$ for $r<\rho_{0}$ lies entirely above the corresponding part of $\Gamma_{2}$. Thus at $u=\beta_{U} \Gamma_{1}$ has inclination angle greater than $\pi / 2$ while $\Gamma_{2}$ has inclination angle less than $\pi / 2$. Also, at $r=\rho_{0}$ the lower part of $\Gamma_{1}$ is above the lower part of $\Gamma_{2}$. We wish to show that $\Gamma_{1}$ remains above $\Gamma_{2}$ for all $r>\rho_{0}$. If this were not the case, the two curves would have to cross somewhere below $u=\beta_{U}$. At the point of crossing, the inclination angle of $\Gamma_{1}$ would have to be less than that of $\Gamma_{2}$. Since at $u=\beta_{U}$ this inequality was reversed, there must be a value of $u$ between $\beta_{U}$ and the value of $u$ 


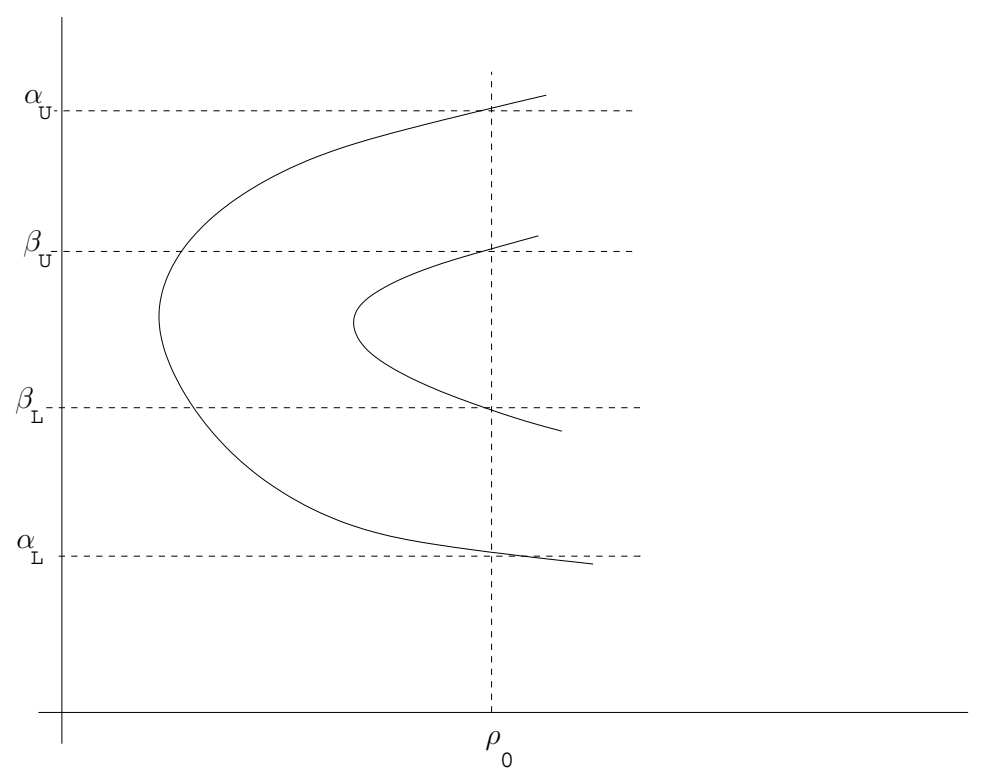

FIG. 9. Case 1.

at the point of crossing at which both curves have the same inclination angle. But by Siegel's uniqueness theorem [10], this would imply that $\Gamma_{1}$ and $\Gamma_{2}$ are identical. Hence they cannot cross, and the lower part of $\Gamma_{1}$ lies above the lower part of $\Gamma_{2}$ for all $r>\rho_{0}$. Thus $\left|B_{1}\right|>\left|B_{2}\right|$.

Now we wish to show that $\left|A_{1}\right|<\left|A_{2}\right|$. Let $\Gamma_{1}^{\prime}$ be the rigid translation of $\Gamma_{1}$ downward by $\alpha_{U}-\beta_{U}$. Then we wish to show that $\Gamma_{1}^{\prime}$ for $r \leq \rho_{0}$ is contained in the region bounded by $\Gamma_{2}$ and $r=\rho_{0}$. We see that $\Gamma_{1}^{\prime}$ and $\Gamma_{2}$ are tangent at $\beta_{U}$. Locally they are both functions $u(r)$ so we consider the second derivative. We compute (using the above differential equations):

$$
\frac{d^{2} u}{d r^{2}}=\frac{1}{\cos ^{2} \phi} \frac{r u+\cos \phi}{-r \cos \phi} .
$$

Since $\phi$ and $r$ are equal for both curves but $u$ is greater for $\Gamma_{1}^{\prime}$ (the differential equation used in evaluating derivatives for $\Gamma_{1}^{\prime}$ uses $u$ values on $\Gamma_{1}$ ), it follows that on $\Gamma_{1}^{\prime}$ we have a smaller (negative) value for $d^{2} u / d r^{2}$, and, hence, for $r$ slightly smaller than $\rho_{0}, \Gamma_{1}^{\prime}$ lies below $\Gamma_{2}$ and has a greater inclination angle.

So we know $\Gamma_{1}^{\prime}$ starts "inside" $\Gamma_{2}$, and now we must ask if it can ever leave. Suppose $\Gamma_{1}^{\prime}$ leaves on the upper portion of $\Gamma_{2}$. Then at the point of leaving $\Gamma_{1}^{\prime}$ has a smaller inclination angle than $\Gamma_{2}$ and thus for some $r$ between $\rho_{0}$ and the radius at the point of leaving, $\Gamma_{1}^{\prime}$ and $\Gamma_{2}$ have the same inclination angle. Thus $\Gamma_{1}$ and $\Gamma_{2}$ have the same inclination angle at that radius. But since this radius is smaller than $\rho_{0}$, this contradicts our choice of $\rho_{0}$ as the smallest radius at which the two curves have the same inclination angle. Thus $\Gamma_{1}^{\prime}$ cannot leave across the 

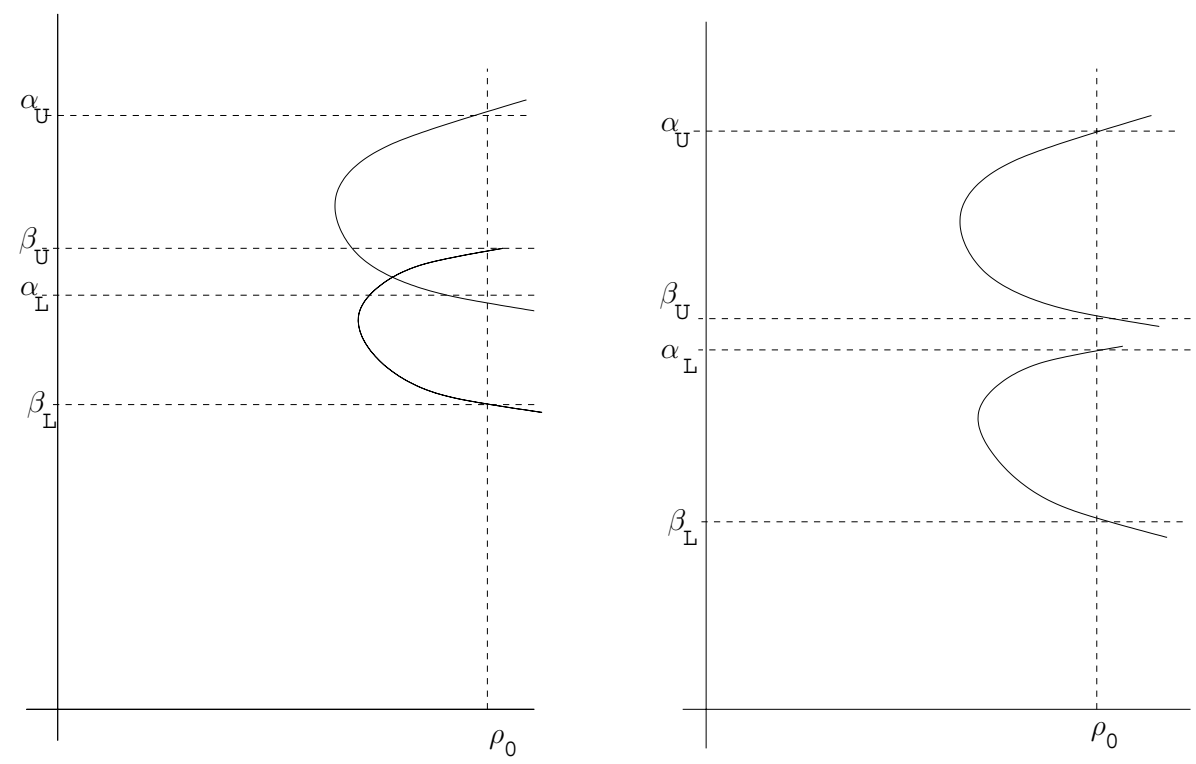

FIG. 10. Case 2, the two cases.

upper portion of $\Gamma_{2}$.

Now it remains to consider whether $\Gamma_{1}^{\prime}$ can leave across the lower portion of $\Gamma_{2}$ for $r \leq \rho_{0}$. If it did, then it could not return since if it did we would again have a radius smaller than $\rho_{0}$ at which the curves have equal inclination angles as in the previous paragraph. So a necessary condition for $\Gamma_{1}^{\prime}$ to "escape" is $\alpha_{U}-\alpha_{L}>\beta_{U}-\beta_{L}$. We prove that this cannot happen. Observe that $\alpha_{U}-\alpha_{L}=$ $\left(\alpha_{U}-T\left(\sigma_{1}\right)\right)+\left(T\left(\sigma_{1}\right)-\alpha_{L}\right)$ and $\beta_{U}-\beta_{L}=\left(\beta_{U}-T\left(\sigma_{2}\right)\right)+\left(T\left(\sigma_{2}\right)-\beta_{L}\right)$. So first we compute

$$
\begin{aligned}
& \alpha_{U}-T\left(\sigma_{1}\right)=\int_{\phi_{0}}^{\pi / 2} \frac{r_{1} \sin \phi}{r_{1} u_{1}+\sin \phi} d \phi \\
& \beta_{U}-T\left(\sigma_{2}\right)=\int_{\phi_{0}}^{\pi / 2} \frac{r_{2} \sin \phi}{r_{2} u_{2}+\sin \phi} d \phi .
\end{aligned}
$$

At $\phi_{0}$, we see that $\frac{\rho_{0} \sin \phi_{0}}{\rho_{0} u_{2}+\sin \phi_{0}}$, the integrand corresponding to $\Gamma_{2}$, is greater than $\frac{\rho_{0} \sin \phi_{0}}{\rho_{0} u_{1}+\sin \phi_{0}}$, the integrand corresponding to $\Gamma_{1}$. We claim that this inequality holds through the interval of integration. If it did not there would be some $\phi$ at which $\frac{r_{1} \sin \phi}{r_{1} u_{1}+\sin \phi}=\frac{r_{2} \sin \phi}{r_{2} u_{2}+\sin \phi}$ which is equivalent to $\left(u_{2}-u_{1}\right) r_{1} r_{2}=\left(r_{2}-r_{1}\right) \sin \phi$. But the left side of this last equation is negative throughout the interval while the right side is positive. Thus the integrands can never be equal and the previous inequality holds throughout the interval. This shows that $\beta_{U}-T\left(\sigma_{2}\right)>\alpha_{U}-T\left(\sigma_{1}\right)$. 
Now we compute

$$
\begin{aligned}
& T\left(\sigma_{1}\right)-\alpha_{L}=\int_{\pi / 2}^{\theta_{1}} \frac{r_{1} \sin \phi}{r_{1} u_{1}+\sin \phi} d \phi \\
& T\left(\sigma_{2}\right)-\beta_{L}=\int_{\pi / 2}^{\theta_{2}} \frac{r_{2} \sin \phi}{r_{2} u_{2}+\sin \phi} d \phi,
\end{aligned}
$$

where $\theta_{1}$ and $\theta_{2}$ are the inclination angles at which the lower portions of $\Gamma_{1}$ and $\Gamma_{2}$ respectively intersect $r=\rho_{0}$. Since $\sigma_{1}>\sigma_{2}$ it follows that the inclination angle of $\Gamma_{1}$ at $\sigma_{1}, \pi / 2$, is less than the corresponding angle for $\Gamma_{2}$. Since $\rho_{0}$ is the smallest radius at which the two can have the same inclination angle, this is true for all $r<\rho_{0}$ and hence $\theta_{2} \geq \theta_{1}$. Further, the same argument as in the previous set of integrals shows that the integrand corresponding to $\Gamma_{2}$ is again greater that that corresponding to $\Gamma_{1}$. Since both integrands are positive and the interval of integration corresponding to $\Gamma_{2}$ contains the interval corresponding to $\Gamma_{1}$ it follows that $T\left(\sigma_{2}\right)-\beta_{L}>T\left(\sigma_{1}\right)-\alpha_{L}$.

Combining the above results shows that $\alpha_{U}-\alpha_{L}>\beta_{U}-\beta_{L}$. As mentioned, this proves that $\Gamma_{1}^{\prime}$ cannot "escape" through the lower portion of $\Gamma_{2}$ and thus cannot escape at all. Since $\Gamma_{1}^{\prime}$ for $r<\rho_{0}$ is contained in the region bounded by $\Gamma_{2}$ and $r=\rho_{0}$, it follows that the volume obtained by rotating that portion of $\Gamma_{2}$ is greater than the corresponding volume obtained from $\Gamma_{1}^{\prime}$. But $\Gamma_{1}^{\prime}$ gives the same volume as $\Gamma_{1}$ since they merely differ by a $u$ translation. Thus we have shown that $\left|A_{1}\right|<\left|A_{2}\right|$.

The above results yield the inequality $\left|B_{1}\right|-\left|A_{1}\right|>\left|B_{2}\right|-\left|A_{2}\right|$. However, Lemma 3.1 tells us that $\left|B_{1}\right|-\left|A_{1}\right|=2 \pi \rho_{0} \sin \phi_{0}=\left|B_{2}\right|-\left|A_{2}\right|$. Thus we have our contradiction, and then the theorem is proven.

For the solutions guaranteed by Theorem 3.1 we will now write $u=u(\psi, \bar{\psi}, \bar{r}, \kappa)$, $r=r(\psi, \bar{\psi}, \bar{r}, \kappa)$. These were defined by the equation $\bar{r}=r(\bar{\psi}, \bar{\psi}, \bar{r}, \kappa)$. Similarly for those guaranteed by Theorem 3.2 we will write $u=u_{e}(\phi, \bar{\phi}, \bar{r}, \kappa), r=r_{e}(\phi, \bar{\phi}, \bar{r}, \kappa)$. By definition $\bar{r}=r_{e}(\bar{\phi}, \bar{\phi}, \bar{r}, \kappa)$.

\section{Existence of a floating drop}

We consider a finite volume of fluid of density $\rho_{1}$ resting on an infinite reservoir of a fluid of density $\rho_{2}$ below another infinite reservoir of a fluid of density $\rho_{0}$. We will assume that $\rho_{0}<\rho_{1}<\rho_{2}$.

We will take as given the surface tensions $\sigma_{01}, \sigma_{02}, \sigma_{12}$ at a contact line between the three fluids, the subscripts indicate that the force acts along the interface between the indicated fluids. The corresponding capillary constants are defined 
by

$$
\begin{aligned}
& \kappa_{01}=\left(\rho_{1}-\rho_{0}\right) g / \sigma_{01}, \\
& \kappa_{02}=\left(\rho_{2}-\rho_{0}\right) g / \sigma_{02}, \\
& \kappa_{12}=\left(\rho_{2}-\rho_{1}\right) g / \sigma_{12} .
\end{aligned}
$$

As we have seen minimization of energy implies that the three surfaces satisfy the equations

$$
\begin{aligned}
M u & =\kappa_{01} u+\lambda / \sigma_{01}, \\
M v & =\kappa_{12} v-\lambda / \sigma_{12}, \\
M w & =\kappa_{02} w
\end{aligned}
$$

where $\lambda$ is a constant which must be determined as a part of the solution of the problem. By force balance (cf. Section 2) $0 \leq \gamma_{01}, \gamma_{02}, \gamma_{12} \leq \pi$, and

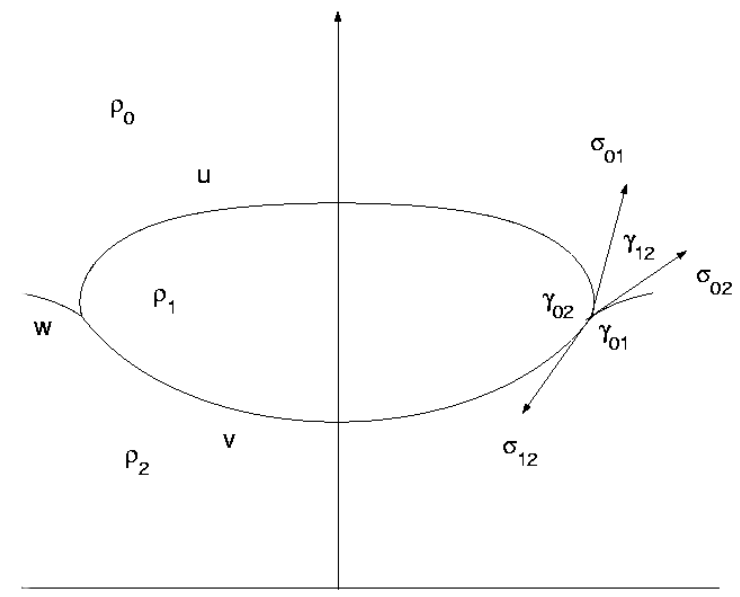

FIG. 11. Contact angles and surface tensions.

$$
\begin{aligned}
& \gamma_{02}=\pi-\arccos \left(\frac{\sigma_{01}^{2}+\sigma_{12}^{2}-\sigma_{02}^{2}}{2 \sigma_{01} \sigma_{12}}\right), \\
& \gamma_{01}=\pi-\arccos \left(\frac{\sigma_{12}^{2}+\sigma_{02}^{2}-\sigma_{01}^{2}}{2 \sigma_{12} \sigma_{02}}\right)
\end{aligned}
$$

(of course $\gamma_{12}=2 \pi-\gamma_{01}-\gamma_{02}$ ), or, equivalently,

$$
\frac{\sin \gamma_{01}}{\sigma_{01}}=\frac{\sin \gamma_{02}}{\sigma_{02}}=\frac{\sin \gamma_{12}}{\sigma_{12}} \text {. }
$$

We note that $\sigma_{01} \kappa_{01}+\sigma_{12} \kappa_{12}=\sigma_{02} \kappa_{02}$. We will fix the radius $\bar{r}$ at which the three surfaces meet, and let $\bar{\psi}$ be the angle that the tangent to $v$ at $\bar{r}$ makes with the horizontal. 


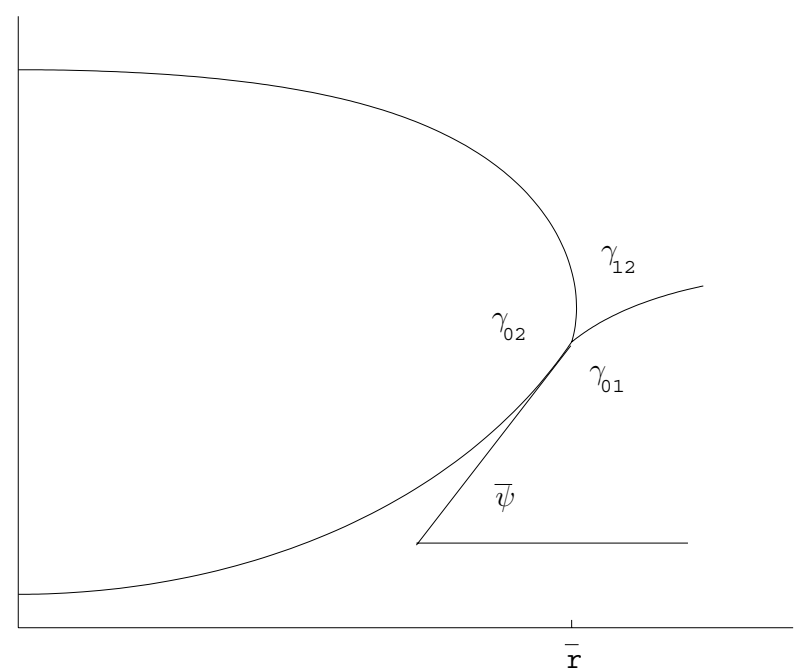

FIG. 12. Inclination angle at the prescribed radius.

We must have $0<\bar{\psi}<\gamma_{02}$. If this were not the case then the drop would be "tipped" up too far and the surface $u$ would be inclined instead of beginning at a zero slope and decreasing. In fact if $\bar{\psi}=\gamma_{02}$, then the fact that the supplement of the sum of $\gamma_{02}$ and the complement of $\bar{\psi}$ is equal to $\pi / 2-\gamma_{02}+\bar{\psi}$ implies this angle is $\pi / 2$. Stated differently, the slope at $\bar{r}$ is zero, and thus the surface $u$ is flat, and any larger angle $\bar{\psi}$ should be excluded from our consideration. This also gives us our first relation between the different boundary conditions. If we have $\bar{\psi}$ for the surface $v$, then for the surface $u \gamma_{02}-\bar{\psi}$ is the angle downward from horizontal. There are several possibilities for the outer surface: $w$ slopes down to the intersection point, $w$ slopes up to the intersection point, and one case of each where $w$ is curled over. The last two cases may seem physically unlikely, but are included for completeness.

There are further geometric constraints. They do not play an explicit role in what follows, but they are included for completeness. We have two cases for $0 \leq \gamma_{02}<\pi / 2$ : if $\gamma_{02} \leq \gamma_{12}$ then $w$ slopes down, and if not then $w$ slopes up. However if we consider $\gamma_{02}>\pi / 2$, the situation is more complicated. We break this into two cases. If $\bar{\psi} \leq \pi / 2$, the interface is below the middle of the drop, and if $\bar{\psi}>\pi / 2$ the interface is above the middle of the drop. There are three configurations contained in each case. First we consider $\bar{\psi} \leq \pi / 2$. If $0 \leq \bar{\psi} \leq$ $\left(\pi / 2-\gamma_{01}\right)$ then $w$ is folded over and sloping down. If $\left(\pi / 2-\gamma_{01}\right) \leq \bar{\psi} \leq\left(\pi-\gamma_{01}\right)$ then $w$ is merely sloping down. If $\left(\pi-\gamma_{01}\right) \leq \bar{\psi} \leq \gamma_{02}$ then $w$ is sloping up. For the second case, we have $\bar{\psi}>\pi / 2$. If $0 \leq \bar{\psi} \leq\left(\pi-\gamma_{01}\right)$ then $w$ is sloping down. If $\left(\pi-\gamma_{01}\right) \leq \bar{\psi} \leq\left(3 \pi / 2-\gamma_{01}\right)$, then $w$ slopes up. If $\left(3 \pi / 2-\gamma_{01}\right) \leq \bar{\psi} \leq\left(\pi-\gamma_{01}\right)$ then $w$ is folded over, sloping up. 
We can match the top and the bottom surfaces, $u$ and $v$, at $\bar{r}$ by eliminating the Lagrange multiplier $\lambda$. Define $U$ and $V$ by $u=-U-\lambda / \kappa_{01} \sigma_{01}$ and $v=$ $V+\lambda / \kappa_{12} \sigma_{01}$. So that $M U=\kappa_{01} U, M V=\kappa_{12} V$. Using the notation introduced at the end of section 3 , let $\bar{U}=u\left(\gamma_{02}-\bar{\psi}, \gamma_{02}-\bar{\psi}, \bar{r}, \kappa_{02}\right), \bar{V}=u\left(\bar{\psi}, \bar{\psi}, \bar{r}, \kappa_{12}\right)$.

Then $u=v$ at $r=\bar{r}$ implies that

$$
\lambda=-[\bar{U}+\bar{V}] \frac{\kappa_{01} \sigma_{01} \kappa_{12} \sigma_{12}}{\kappa_{01} \sigma_{01}+\kappa_{12} \sigma_{12}} .
$$

If we put this back into the equations that defined $U$ and $V$ we get for the common height of $u$ and $v$ at $\bar{r}$

$$
\frac{\kappa_{12} \sigma_{12} \bar{V}-\kappa_{01} \sigma_{01} \bar{U}}{\kappa_{01} \sigma_{01}+\kappa_{12} \sigma_{12}} .
$$

We can also write for the height of $\bar{w}$ at $\bar{r}, w=u_{e}\left(\gamma_{01}+\bar{\psi}, \gamma_{02}+\bar{\psi}, \bar{r}, \kappa_{02}\right)$ since $\bar{\phi}=\gamma_{01}+\bar{\psi}$.

The surfaces $U$ and $V$ are capillary surfaces or inverted sessile drops as described in Theorem 3.1. $V$ makes the angle $\bar{\psi}$ at $\bar{r}$, and $U$ makes the angle $\gamma_{02}-\bar{\psi}$.

We write the difference of $u$ and $w$ at $\bar{r}$ as

and vary $\bar{\psi}$.

$$
F(\bar{\psi})=\frac{\kappa_{12} \sigma_{12} \bar{V}-\kappa_{01} \sigma_{01} \bar{U}}{\kappa_{01} \sigma_{01}+\kappa_{12} \sigma_{12}}-\bar{w}
$$

By looking at limiting cases we see that $F(0)<0$, since then $\bar{V} \equiv 0, \bar{U}>0$, and $\bar{w} \geq 0$. Similarly, $F\left(\gamma_{02}\right)>0$, since then $\bar{V}>0, \bar{U} \equiv 0$, and $\bar{w} \leq 0$. (we have used here that fact that $w$ is zero at infinity.)

We have proven

Theorem 4.1. For each $\bar{r}$, there is a drop in which the three surfaces meet at radius $\bar{r}$.

For $0<\gamma_{02}<\pi / 2$ all three surfaces $u, v, w$ must be graphs. In this case we have

Theorem 4.2. Suppose that $0<\gamma_{02}<\pi / 2$. Then there is a drop of volume $\mathcal{V}$ for every prescribed $\mathcal{V}>0$.

Proof. First we observe that the results of Siegel [10] and the argument at the beginning of the proof of Theorem 5.3 show that $\partial F / \partial \bar{\psi}>0$. Then there is a unique $\bar{\psi}(\bar{r})$ satisfying $F(\bar{\psi})=0$ for each $\bar{r}>0$, and $\bar{\psi}(\bar{r})$ depends continuously on $\bar{r}$.

The volume can be written as the sum of volumes of two sessile drops ([1, page 40] for the volume of a sessile drop),

$$
\begin{aligned}
\mathcal{V} & =\mathcal{V}_{1}+\mathcal{V}_{2} \\
\mathcal{V}_{1} & =\pi \bar{r}\left(\bar{r} V-2 \sin \bar{\psi} / \kappa_{12}\right), \\
\mathcal{V}_{2} & =\pi \bar{r}\left(\bar{r} U-2 \sin \left(\gamma_{02}-\bar{\psi}\right) / \kappa_{01}\right)
\end{aligned}
$$


We can estimate $\mathcal{V}$ from below by considering two cases, $\bar{\psi} \geq \gamma_{02} / 2, \bar{\psi}<\gamma_{02} / 2$. In the first case

$$
\begin{aligned}
\mathcal{V}_{1} & \geq \pi \bar{r}\left(\bar{r} u\left(\gamma_{02} / 2, \gamma_{02} / 2, \bar{r}, \kappa_{12}\right)-2 / \kappa_{12}\right) \\
& \geq \pi \bar{r}\left(\bar{r} \sqrt{\frac{2}{\kappa_{12}}\left(1-\cos \left(\frac{\gamma_{02}}{2}\right)\right)}-\frac{2}{\kappa_{12}}\right)
\end{aligned}
$$

where the second inequality follows from [10, Theorem 7]. In the second case

$$
\mathcal{V}_{2} \geq \pi \bar{r}\left(\bar{r} \sqrt{\frac{2}{\kappa_{01}}\left(1-\cos \left(\frac{\gamma_{02}}{2}\right)\right)}-\frac{2}{\kappa_{01}}\right) \text {. }
$$

Combining these

$$
\mathcal{V} \geq \pi \bar{r}\left(\bar{r} \sqrt{\frac{2}{\kappa_{1}}\left(1-\cos \left(\frac{\gamma_{02}}{2}\right)\right)}-\frac{2}{\kappa_{2}}\right)
$$

where $\kappa_{1}=\max \left(\kappa_{01}, \kappa_{12}\right), \kappa_{2}=\min \left(\kappa_{01}, \kappa_{12}\right)$. It follows that $\mathcal{V} \rightarrow \infty$ as $\bar{r} \rightarrow \infty$. Also $\mathcal{V} \leq 2 \pi \bar{r}^{2} u\left(\pi / 2, \pi / 2, \bar{r}, \kappa_{1}\right)$, which is $\mathcal{O}(1 / \bar{r})[1, \mathrm{p} 27]$, so $\mathcal{V}=\mathcal{O}(\bar{r})$ as $\bar{r} \rightarrow 0$. The theorem follows.

A partial analogue of Theorem 4.1 can be given for a finite circular cylindrical container with an axisymmetric drop lying on the symmetry axis. The role of $w$ is taken over by a surface over an annular domain. We consider here only the case in which the annular surface is a graph. The only property of $w$ which was used in showing that $F(\bar{\psi})$ changes sign is that $w^{\prime}(\bar{r}) \leq 0$ implies that $w(\bar{r}) \geq 0$ and $w^{\prime}(\bar{r}) \geq 0$ implies that $w(\bar{r}) \leq 0$. Assuming that the contact angle at the container boundary is in $[0, \pi / 2]$, this follows from the basic comparison theorem for capillary surfaces, [1, Theorem 5.1], if $w$ is compared with zero. The general case requires detailed properties of capillary surfaces over annular domains which will not be discussed here.

We now give some asymptotic results for small drops.

Lemma 4.1. For a capillary surface determined by $\bar{r}$ and $\bar{\psi}$ (as in Theorem 3.1),

$$
u(\bar{\psi}, \bar{\psi}, \bar{r}, \kappa):=\tilde{V}=\frac{2 \sin \bar{\psi}}{\kappa \bar{r}}+\mathcal{O}(\bar{r}), \quad \text { as } \bar{r} \rightarrow 0,
$$

uniformly for $\bar{\psi}$ bounded away from $\pi$.

Proof. Case 1. $\bar{\psi} \leq \pi / 2$. This follows from [1,2.41] and [10].

Case 2. $\pi / 2 \leq \bar{\psi} \leq \pi-\epsilon$ for some $\epsilon>0$. First

$$
\tilde{V}=2 / \kappa R+\mathcal{O}(R) \quad \text { as } R \rightarrow 0
$$

by $[1$, p. 55]. Then

$$
\bar{r}=R \sin \bar{\psi}+\mathcal{O}\left(R^{3}\right)
$$

by $[1$, pp. 52-53, 3.46, 3.53b, 3.54c]. Combining these gives the result. 
Definition 4.1. For $0<\gamma_{02}<\pi$ define $\gamma_{c}$ as the unique root of

$$
\frac{\sin \psi}{\sin \left(\gamma_{02}-\psi\right)}=\frac{\sigma_{01}}{\sigma_{12}}
$$

in $\left(0, \gamma_{02}\right)$.

This makes sense since $g(\psi)=\sin \psi / \sin \left(\gamma_{02}-\psi\right)$ is strictly increasing on $\left(0, \gamma_{02}\right)$ and $g(0)=0, \lim _{\psi \rightarrow \gamma_{02}-} g(\psi)=\infty$.

Theorem 4.3. 1. Suppose that $0<\gamma_{02}<\pi$. Then for $\epsilon>0$ there is an $r_{0}>0$ such that $F(\bar{\psi}) \neq 0$ on $\left[0, \gamma_{c}-\epsilon\right] \cup\left[\gamma_{c}+\epsilon, \pi\right]$ for $0<r \leq r_{0}$.

2. Suppose that $\gamma_{02}=\pi$. Then for $\epsilon>0$ there is an $\bar{r}_{0}>0$ such that $F(\bar{\psi}) \neq 0$ on $[\epsilon, \pi-\epsilon]$ for $0<r \leq r_{0}$.

Proof. Lemma 4.1 implies that

$$
\bar{V}=\frac{2 \sin \bar{\psi}}{\kappa_{12} \bar{r}}+\mathcal{O}(\bar{r}) \quad \text { as } \bar{r} \rightarrow 0
$$

and

$$
\bar{U}=\frac{2 \sin \left(\gamma_{02}-\bar{\psi}\right)}{\kappa_{01} \bar{r}}+\mathcal{O}(\bar{r}) \quad \text { as } \bar{r} \rightarrow 0 .
$$

Using results of Turkington [12] and Vogel [13] $w=\mathcal{O}(\bar{r} \ln \bar{r})$ as $\bar{r} \rightarrow 0$, so

$$
\begin{aligned}
F(\bar{\psi}) & =\frac{\kappa_{12} \sigma_{12} \frac{2 \sin \bar{\psi}}{\kappa_{12} \bar{r}}-\kappa_{01} \sigma_{01} \frac{2 \sin \left(\gamma_{02}-\bar{\psi}\right)}{\kappa_{01} \bar{r}}}{\kappa_{01} \sigma_{01}+\kappa_{12} \sigma_{12}}+\mathcal{O}(\bar{r} \ln \bar{r}) \\
& =\frac{2}{\kappa_{01} \sigma_{01}+\kappa_{12} \sigma_{12}} \frac{1}{\bar{r}}\left(\sigma_{12} \sin \bar{\psi}-\sigma_{01} \sin \left(\gamma_{02}-\bar{\psi}\right)\right)+\mathcal{O}(\bar{r} \ln \bar{r}) \quad \text { as } \bar{r} \rightarrow 0
\end{aligned}
$$

This theorem shows that as $\bar{r}$ tends to zero, a $\bar{\psi}$ such that $F(\bar{\psi})=0$ tends to $\psi_{c}$ in case 1 and either to 0 or $\pi$ in case 2 .

\section{The floating bubble}

We give a separate treatment to a limiting case of the floating drop in which $\rho_{0}=\rho_{1}, \sigma_{12}=\sigma_{02}:=\sigma$ and $\sigma_{01}=2 \sigma$. We may imagine a bubble of the top fluid, e.g. air, which is bounded on top by a thin film of the bottom fluid. The contact angles are $\gamma_{02}=\pi, \gamma_{01}=0, \gamma_{12}=\pi$. The differential equations become

$$
\begin{aligned}
M u & =\lambda / 2 \sigma, \\
M v & =\kappa v-\lambda / \sigma, \\
M w & =\kappa w,
\end{aligned}
$$


where $\kappa=\left(\rho_{2}-\rho_{0}\right) g / \sigma$, and we can explicitly solve the equation for $u, \sin \psi=$ $-\lambda r / 4 \sigma$ so that

$$
\lambda=-4 \sigma \sin \bar{\psi} / \bar{r}
$$

The top surface is a section of a sphere, and $v=V+\lambda / \kappa \sigma$ where $M V=\kappa V$. The equation (17) guarantees that the sphere meets the lower surface at $\bar{r}$, and we write for $v-w$ at $\bar{r}$

$$
F(\bar{\psi})=\bar{V}-\frac{4 \sin \bar{\psi}}{\kappa \bar{r}}-\bar{w} .
$$

where $\bar{V}=u(\bar{\psi}, \bar{\psi}, \bar{r}, \kappa)$ and $\bar{w}=u_{e}(\bar{\phi}, \bar{\phi}, \bar{r}, \kappa)$ with $\bar{\phi}=\bar{\psi}$ (since $\gamma_{01}=0$.)

Since $F(0)=-\bar{w}<0$ and $F(\pi)=\bar{V}>0$ we can deduce existence of a bubble as before. But we can do better. Using [10, Theorem 7],

$$
u\left(\frac{\pi}{2}, \frac{\pi}{2}, \bar{r}, \kappa\right):=\bar{V}\left(\bar{r}, \frac{\pi}{2}\right)<\frac{1}{\kappa \bar{r}}+\left(\frac{2}{\kappa}+\frac{1}{(\kappa \bar{r})^{2}}\right)^{1 / 2}
$$

and [10, Theorem 14]

$$
u_{e}\left(\frac{\pi}{2}, \frac{\pi}{2}, \bar{r}, \kappa\right):=\bar{w}\left(\bar{r}, \frac{\pi}{2}\right)>-\frac{1}{\kappa \bar{r}}+\left(\frac{2}{\kappa}+\frac{1}{(\kappa \bar{r})^{2}}\right)^{1 / 2}
$$

so $F(\pi / 2)<-2 / \kappa \bar{r}$ and there is a solution for $\bar{\psi} \in(\pi / 2, \pi)$. This does not prohibit a sign change for $F$ in $(0, \pi / 2)$, but we have

Theorem 5.1. $F(\bar{\psi})<0$ for $\bar{\psi} \in(0, \pi / 2)$.

Proof. From [10, Theorem 7]

$$
u(\bar{\psi}, \bar{\psi}, \bar{r}, \kappa):=\bar{V}(\bar{r}, \bar{\psi})<\frac{\sin \bar{\psi}}{\kappa \bar{r}}+\sqrt{\frac{2}{\kappa}(1-\cos \bar{\psi})+\left(\frac{\sin \bar{\psi}}{\kappa \bar{r}}\right)^{2}}
$$

and [10, Theorem 14] together with the decrease of the exterior surfaces with respect to contact angle implies

$$
u_{e}(\bar{\psi}, \bar{\psi}, \bar{r}, \kappa):=\bar{w}(\bar{r}, \bar{\psi}) \geq T(\sigma) \geq-\frac{1}{\kappa \sigma}+\sqrt{\frac{2}{\kappa}+\left(\frac{1}{\kappa \sigma}\right)^{2}} .
$$

Since $w$ bends over for $\bar{\psi} \in(0, \pi / 2)$, [13, equation 2.9] implies

$$
(r \sin \phi)_{r}=-\kappa r w
$$

on the top, and integrating from $\sigma$ to $r$,

$$
\sigma-r \sin \phi=\kappa \int_{\sigma}^{r} \rho w d \rho>0
$$

so that $\sigma>r \sin \psi$. Using $T(\sigma) \leq w$ we can estimate the integral to obtain

$$
\frac{\sigma-r \sin \psi}{\kappa} \geq T(\sigma) \frac{r^{2}-\sigma^{2}}{2}
$$


i.e.

$$
r^{2}-\sigma^{2} \leq 2 \frac{\sigma-r \sin \psi}{\kappa T(\sigma)} .
$$

We proceed by breaking up the interval into two parts. Consider first $[\pi / 5, \pi / 2)$. The above estimates imply

$$
F(\bar{\psi})<-\frac{3 \sin \bar{\psi}}{\kappa \bar{r}}+\frac{1}{\kappa \sigma}+\sqrt{\frac{2}{\kappa}(1-\cos \bar{\psi})+\left(\frac{\sin \bar{\psi}}{\kappa \bar{r}}\right)^{2}}-\sqrt{\frac{2}{\kappa}+\left(\frac{1}{\kappa \sigma}\right)^{2}},
$$

and $\sigma<r$ implies the difference of square roots is negative. The sum of the first two terms is negative if $\sigma \geq \bar{r} / 3 \sin \bar{\psi}$, and this is implied by $\bar{r} \sin \bar{\psi} \geq \bar{r} / 3 \sin \bar{\psi}$, i.e. $\sin \bar{\psi} \geq 1 / \sqrt{3}$, which is implied by $\bar{\psi} \geq \pi / 5$.

For the interval $(0, \pi / 5)$ we consider two cases depending on the magnitude of $r$. We have

$$
F(\bar{\psi}) \leq \sqrt{\frac{2}{\kappa}(1-\cos \bar{\psi})+\left(\frac{\sin \bar{\psi}}{\kappa \bar{r}}\right)^{2}}-3 \frac{\sin \bar{\psi}}{\kappa \bar{r}} .
$$

The right hand side is nonnegative if

$$
g(\bar{\psi}):=\frac{4 \sin ^{2} \bar{\psi}}{\kappa \bar{r}^{2}}+\cos \bar{\psi} \geq 1 .
$$

Since $g(0) \geq 1$, this inequality holds as long as

$$
g^{\prime}(\bar{\psi})=\frac{8}{\kappa \bar{r}^{2}} \sin \bar{\psi} \cos \bar{\psi}-\sin \bar{\psi} \geq 0
$$

i.e. $\kappa \bar{r}^{2} / 9 \leq \cos \bar{\psi}$ which simplifies to $\bar{r} \leq \sqrt{8 \cos \bar{\psi} / \kappa}$.

This is our first case.

Since (23) holds on the top, equations (5) imply ([10], equation (27))

$$
-(\cos \phi)_{w}+\frac{1}{r} \sin \phi=-w \kappa
$$

there. For $\sigma \leq \rho<r$, integrating from $w(\rho)$ to $w$ yields

$$
-\cos \phi(r)+\cos \phi(\rho)+\int_{w(\rho)}^{w(r)} \frac{\sin \phi}{r} d u=-\kappa \frac{w^{2}(r)-w^{2}(\rho)}{2},
$$

and, letting $\rho \rightarrow \sigma$,

$$
-\kappa\left[w^{2}(r)-T^{2}(\sigma)\right]=-2 \cos \psi(r)+2 \int_{T(\sigma)}^{w} \frac{\sin \phi}{r} d u .
$$

Since $\sin \phi / r \leq 1 / \sigma$

$$
\kappa\left[w^{2}(r)-T^{2}(\sigma)\right] \geq 2 \cos \phi(r)-2 \frac{w(r)-T(\sigma)}{\sigma},
$$


which implies

$$
w \geq-\frac{1}{\sigma \kappa}+\sqrt{\frac{1}{\sigma^{2} \kappa^{2}}+\frac{2}{\kappa} \cos \phi+\frac{2}{\sigma \kappa} T+T^{2}} .
$$

With this sharpened lower bound for $w$

$$
F(\bar{\psi}) \leq-\frac{3 \sin \bar{\psi}}{\kappa \bar{r}}+\sqrt{\frac{2}{\kappa}(1-\cos \bar{\psi})+\frac{\sin ^{2} \bar{\psi}}{\kappa^{2} \bar{r}^{2}}}+\frac{1}{\sigma \kappa}+\sqrt{\frac{1}{\sigma^{2} \kappa^{2}}+\frac{2}{\kappa} \cos \bar{\psi}+\frac{2}{\sigma \kappa} T+T^{2}}
$$

We estimate $F(\bar{\psi})$ from above with the following steps. First replace the first square root with the sum of the square roots of the two terms to obtain $F(\bar{\psi}) \leq$ $h(\bar{\psi})$. We can rewrite $h(\bar{\psi})<0$ as

$$
\sqrt{\frac{1}{\sigma^{2} \kappa^{2}}+\frac{2}{\kappa} \cos \bar{\psi}+\frac{2}{\sigma \kappa} T+T^{2}}>\frac{1}{\sigma \kappa}+\sqrt{\frac{2(1-\cos \bar{\psi})}{\kappa}}-\frac{2 \sin \bar{\psi}}{\kappa \bar{r}} .
$$

Square both sides of this inequality and use again $\sin \bar{\psi} / \bar{r} \leq 1 / \sigma$ to obtain

$$
\frac{2}{\kappa}(2 \cos \bar{\psi}-1)+\frac{2}{\sigma \kappa} T+T^{2}>\frac{2}{\sigma \kappa} \sqrt{\frac{2}{\kappa}(1-\cos \bar{\psi})}-\frac{4 \sin \bar{\psi}}{\kappa \bar{r}} \sqrt{\frac{2}{\kappa}(1-\cos \bar{\psi})} .
$$

On $(0, \pi / 5)$ this is implied by

$$
\frac{2}{\sigma \kappa} T+T^{2}>\frac{2}{\sigma \kappa} \sqrt{\frac{2}{\kappa}(1-\cos \bar{\psi})} .
$$

The estimate (22) implies that

$$
\frac{2}{\sigma \kappa} T+T^{2}>\frac{2}{\kappa}
$$

so that this last inequality is implied by

$$
\sigma>\sqrt{\frac{2}{\kappa}(1-\cos \bar{\psi})} .
$$

It remains to show that either (41) or $\bar{r} \leq \sqrt{8 \cos \bar{\psi} / \kappa}$ holds. We have shown that $r^{2}-\sigma^{2} \leq 2(\sigma-r \sin \phi) / \kappa T$ on the top of the graph of $w$. Suppose that $r>\sqrt{8 \cos \bar{\psi} / \kappa}$ and $\sigma \leq \sqrt{2(1-\cos \bar{\psi}) / \kappa}$. Then $r^{2}-\sigma^{2}>2(5 \cos \psi-1) / \kappa$. To get a contradiction we need to show that

$$
5 \cos \bar{\psi}-1 \geq \frac{\sigma-r \sin \bar{\psi}}{T} .
$$

This is implied by

$$
\frac{5 \cos \bar{\psi}-1}{1-\sin \bar{\psi}} \geq \frac{\sigma}{T}
$$


since $\sigma<r$. The left hand side of this inequality is greater than 4 on $(0, \pi / 5)$, so we need only show that $\sigma / T \leq 4$. Using (20) this is implied by

$$
\begin{aligned}
\sigma^{2} & \leq 4\left(-\frac{1}{\kappa}+\sqrt{\frac{2}{\kappa} \sigma^{2}+\frac{1}{\kappa^{2}}}\right) \\
& =4 \frac{\frac{2}{\kappa} \sigma^{2}}{\frac{1}{\kappa}+\sqrt{\frac{2}{\kappa} \sigma^{2}+\frac{1}{\kappa^{2}}}}
\end{aligned}
$$

which is, in turn, implied by $\sigma^{2} \leq 24 / \kappa$. It suffices to consider the largest value of $\sigma$ under consideration, namely $\sigma=\sqrt{2(1-\cos \bar{\psi}) / \kappa}$ and this inequality is satisfied for this choice of $\sigma$. The theorem is proven.

We can also give an asymptotic result for smaller bubbles.

Theorem 5.2. For $\epsilon>0$ there is an $r_{0}>0$ such that $F(\bar{\psi})<0$ on $[\pi / 2, \pi-\epsilon]$ for $r \leq r_{0}$.

Proof. By Lemma 3.1

$$
F(\bar{\psi})=-\frac{2 \sin \bar{\psi}}{\kappa \bar{r}}+\mathcal{O}(\bar{r})-w(\bar{r}, \bar{\psi})
$$

and [10, Theorem 14] implies

$$
\begin{aligned}
F(\bar{\psi}) & \leq-\frac{\sin \bar{\psi}}{\kappa \bar{r}}+\mathcal{O}(\bar{r})-\sqrt{\frac{2}{\kappa}(1+\cos \bar{\psi})+\frac{\sin ^{2} \bar{\psi}}{\kappa \bar{r}}} \\
& \leq-\frac{\sin \bar{\psi}}{\kappa \bar{r}}+\mathcal{O}(\bar{r}) .
\end{aligned}
$$

Finally, we prove uniqueness of the solution for fixed $\bar{r}$. For this is suffices to show that

$$
F(\bar{\psi})=\bar{V}-\frac{4 \sin \bar{\psi}}{\kappa \bar{r}}-\bar{w}
$$

is increasing on $(\pi / 2, \pi)$ since we have shown that $F<0$ in $[0, \pi / 2]$.

Theorem 5.3. For fixed $\bar{r}$ there is exactly one bubble.

Proof. We denote by $\Delta=\kappa r u-\sin \psi$ the denominator in equations (4). In finding the folded over surface $V$ which is the bottom of the bubble $u_{0}$ must be varied so that $r\left(\bar{\psi} ; u_{0}\right)=\bar{r}$. We denote the derivative with respect to $u_{0}$ by $\left({ }^{\cdot}\right)$ as in $[1$, 
chapter 3]. Then, denoting derivatives with respect to $\bar{\psi}$ by ( ) $)^{\prime}$

$$
\begin{aligned}
\frac{d V}{d \bar{\psi}} & =V^{\prime}+\dot{V} u_{0}^{\prime} \\
& =V^{\prime}+\dot{V}\left(\frac{-r^{\prime}}{\dot{r}}\right) \\
& =\frac{r}{\dot{r} \Delta}(\dot{r} \sin \bar{\psi}-\dot{V} \cos \bar{\psi}) \\
& :=\frac{-r}{\dot{r} \Delta} f(\bar{\psi}),
\end{aligned}
$$

where the implicit function theorem has been applied to $r\left(\bar{\psi} ; u_{0}\right)-\bar{r}=0$, and (4) has been used. The function $f(\psi)$ is zero exactly once in $(0, \pi)$ (always in $(\pi / 2, \pi)$ ) and the solution $\psi^{\prime}$ is the contact point of the envelope of the family of solutions of (4) parameterized by $u_{0}$. For $\psi<\psi^{\prime}, f(\psi)>0$, so that for $\bar{\psi}<\psi^{\prime}, d V / d \bar{\psi}>0$, and $F(\bar{\psi})$ is increasing on $\left[\pi / 2, \psi^{\prime}\right)$ since $w(\bar{r}, \bar{\psi})$ decreases as $\bar{\psi}$ increases from $\pi / 2$.

It will suffice for us to show that $g(\bar{\psi}):=V(\bar{\psi})-4 \sin \bar{\psi} / \kappa \bar{r}$ is increasing on $\left(\psi^{\prime}, \pi\right)$.

Let us denote by $\mathcal{V}(\bar{\psi})$ the volume obtained by rotating $V$ around the vertical axis. Then

$$
\mathcal{V}(\bar{\psi})=\pi \bar{r}\left(V \bar{r}-\frac{2}{\kappa} \sin \bar{\psi}\right) .
$$

We claim that $\mathcal{V}(\bar{\psi})$ is increasing in $\bar{\psi}$. Suppose for the moment that we assume this to be true. Then, for $\epsilon>0, \mathcal{V}(\bar{\psi}+\epsilon)>\mathcal{V}(\bar{\psi})$ can be rewritten as

$$
\mathcal{V}(\bar{\psi}+\epsilon)-\mathcal{V}(\bar{\psi})>\frac{2}{\kappa \bar{r}}(\sin (\bar{\psi}+\epsilon)-\sin \bar{\psi}) .
$$

This implies

$$
g(\bar{\psi}+\epsilon)-g(\bar{\psi})>\frac{-2}{\kappa \bar{r}}(\sin (\bar{\psi}+\epsilon)-\sin \bar{\psi})
$$

and the right hand side is positive since $\bar{\psi} \in(\pi / 2, \pi)$.

To see that $\mathcal{V}(\bar{\psi})$ increases for $\bar{\psi} \in(\pi / 2, \pi]$ we need the fact that $\dot{\mathcal{V}}(\bar{\psi})<0$ which was proven by Finn [1, Theorem 3.2]. For fixed $\bar{r}$ increasing $\bar{\psi}$ decreases $u_{0}$ (since $\dot{r}<0$, also proven by Finn). For a smaller value of $u_{0}$ and $\bar{r}, \bar{\psi}$ fixed the point on the graph of $V$ is below and to the right and has bigger volume. Increasing $\bar{\psi}$ will make the volume even bigger.

Corollary 5.1. There is a bubble of every prescribed positive volume.

Proof. We need only invoke estimates analogous to those in the proof of Theorem 4.2 to see that the volume goes to zero as $\bar{r}$ approaches zero and to infinity as $\bar{r}$ goes to infinity. 


\section{Open problems}

There are several questions which we have not been able to answer using our methods.

The simplest and perhaps most vexing one is uniqueness of drops of prescribed volume in the nonparametric case $\left(\gamma_{02}<\pi / 2\right)$. Prescription of the volume is the natural physical condition, and it seems certain that this is true.

It would be desirable to show in general that the equation $F(\bar{\psi} ; \bar{r})=0$ has a continuous branch of solutions for $0<\bar{r}<\infty$. This then implies the existence of a drop with given volume. For bubbles the uniqueness in Theorem 5.3 makes this unnecessary and existence for a given volume is proven.

For small bubbles we have shown that the limiting case is a submerged bubble (Theorem 5.2). This was exhibited in numerical calculations [4]. In the opposite case of large bubbles the limiting case was a hemisphere standing on a flat surface. It would be interesting to prove this.

The uniqueness of a drop of given radius in the case $\pi / 2 \leq \gamma_{02} \leq \pi$ seems plausible in view of the uniqueness for floating bubbles. In this case it may be useful to note that $F(\bar{\psi} ; \bar{r})$ is increasing on the interval $\left[\gamma_{02}-\pi / 2, \pi / 2\right]$. However, computations show that we may have $F<0$ at $\pi / 2$.

The authors are indebted to the referee and to Bob Finn for suggestions which led to considerable improvement in the exposition.

\section{References}

[1] R. Finn, Equilibrium Capillary Surfaces, Springer-Verlag, New York 1986.

[2] S. T. Gibis, Ph. D. Thesis Research Proposal, University of Waterloo, 1989.

[3] S. Hartland and R. W. Hartley, Axisymmetric Fluid-Liquid Interfaces, Elsevier, Amsterdam 1976

[4] Kevin Hemphill, Bubble Development of Known Radius from Capillary Surfaces, M.S. Thesis, Wichita State University, 1999.

[5] W. E. Johnson and L. M. Perko, Interior and exterior boundary value problems from the theory of the capillary tube, Arch. Rational Mech. Anal. 29 (1968), 125-143.

[6] A. D. Myshisis, V. G. Babskit, N. D. Kopachevskit, L. A. Slobozhanin and A. D. Typuptsov, Low-Gravity Fluid Mechanics, Springer-Verlag, New York 1987.

[7] H. M. Princen, Shape of a fluid at a liquid-liquid interface, J. Colloid Science 18 (1963), $178-195$.

[8] H. M. Princen and S. G. Mason, Shape of a fluid-liquid interface I. Extension and test of two-phase theory, J. Colloid Science 20 (1965), 156-172.

[9] H. M. Princen and S. G. Mason, Shape of a fluid-liquid interface II. Theory for threephase systems, J. Colloid Science 20 (1965), 246-266.

[10] D. Siegel, Height estimates for capillary surfaces, Pacific J. Math. 88 (1980), 471-516.

[11] L. A. Slobozhanin, Equilibrium and stability of three capillary fluids with a common line of contact, Izvestiya Akademii Nauk SSSR, Mekhanika Zhidkosti i Gaza 176 (3) (1986), $170-173$.

[12] B. Turkington, Height estimates for exterior problems of capillary type, Pacific J. Math. 88 (1980), 517-540.

[13] T. I. Vogel, Symmetric unbounded liquid bridges, Pacific J. Math. 103 (1982), 205-241. 


\author{
Alan Elcrat \\ Department of Mathematics and Statistics \\ Wichita State University \\ Wichita KA 67260-0033 \\ U.S.A. \\ David Siegel \\ Department of Applied Mathematics \\ University of Waterloo \\ Waterloo, ON N2L 3G1 \\ Canada
}

Robert Neel

Department of Mathematics

Harvard University

U.S.A.

(accepted: May 25, 2003)

To access this journal online:

(20) http://www.birkhauser.ch 